\title{
INSTRUMENTAÇÃO ELETRÔNICA COM METROLOGIA: BASES PARA UMA ABORDAGEM INTEGRADA
}

\author{
Flávio H. Vasconcelos* \\ fvasc@dee.ufmg.br \\ Walace C.Boaventura* \\ wventuraddee.ufmg.br \\ *Departamento de Engenharia Elétrica
Universidade Federal de Minas Gerais Campus - UFMG Pampulha, Belo Horizonte, Brasil \\ *Departamento de Engenharia Elétrica
Universidade Federal de Minas Gerais Campus - UFMG Pampulha, Belo Horizonte, Brasil \\ Elson J.Silva* \\ elson@dee.ufmg.br
}

\begin{abstract}
Metrology and Instrumentation: bases to an integrated approach

Electronic Instrumentation and Metrology are topics that frequently take part of the engineering curriculum. Despite the common background, seldom they are taught under the same point of view and employing a common and non-contrasting pedagogical approach. In this paper it is proposed a coherent framework which can be used to elaborate undergraduate as well as postgraduate course units, integrating both subjects. Subverting the traditional logic, each course unit is composed by a group of learning outcomes - the competences that the student has to demonstrate to be approved. The traditional course syllabus appears as part of the teaching/learning strategy.
\end{abstract}

KEYWORDS: electrical metrology, instrumentation, teaching, student assessment.

\section{RESUMO}

Instrumentação e metrologia estão entre os topicos que devem ser estudados nos cursos de engenharia. A despeito de terem uma relação de complementariedade, quando presentes nos currículos, quase nunca são tratados sob um mesmo ponto de vista e com uma abordagem comum e

\footnotetext{
Artigo submetido em 13/11/2009 (Id.: 01077)

Revisado em 05/04/2010, 03/11/2010

Aceito sob recomendação do Editor Associado Prof. Sebastian Yuri Cavalcanti Catunda
}

não-contrastante. Neste artigo é proposto um arcabouço racional para orientar a elaboração de disciplinas, de graduação ou de pós-graduação, integrando instrumentação eletrônica com metrologia. Partindo de uma lógica oposta à tradicional, a definição da disciplina toma como base o que será avaliado - as competências que estudante tem de adquirir. Os tradicionais conteúdos aparecem em decorrência das estratégias de ensino/aprendizagem, elaboradas para que o aluno possa demonstrar ter adquirido os conhecimentos e habilidades requeridos.

PALAVRAS-CHAVE: instrumentação, metrologia elétrica, ensino por competências, avaliação.

\section{INTRODUÇÃO}

A Instrumentação Eletrônica é compreendida aqui como o ramo da engenharia que projeta, constroi, testa e especifica instrumentos, sistemas e dispositivos que realizam estas medições. Metrologia, na visão expressa neste artigo, é tomada no seu significado literal, como a ciência das medições. Assim, uma complementa a outra.

A análise de currículos dos cursos de engenharia de várias universidades do Brasil e do exterior na área elétrica, permite concluir que em vários existem uma ou mais disciplinas abordando instrumentação. O mesmo não acontece com metrologia. Esta, quando presente, é, muitas vezes, tratada de forma restrita e ultrapassada.

A abordagem dos tópicos instrumentação e metrologia 
em cursos de engenharia de algumas universidades e faculdades brasileiras recebe influências de seus similares de universidades dos Estados Unidos e, com menor frequência, da Europa. É muito comum também que os modelos sejam ou cursos de universidades brasileiras mais conceituadas ou ditado pela tradição da escola.

Em alguns desses cursos percebe-se a existência de disciplinas denominadas "Medidas Elétricas" ou algum outro nome similar. Nestes, em sua maioria, o foco do ensino envolve estudo dos equipamentos para medição de altas tensões e altas correntes e instrumentos eletromêcanicos de medição de tensão, corrente, potência e energia. Osciloscópios são foco de atenção apenas quanto à sua operação. São deixados de lado temas como sensores, eletrônica para condicionamento de sinais, interfaciamento com o sistema computacional, incerteza do resultado da medição, dentre outros. Chama atenção a abordagem inadequada da teoria de erros. Isso é, provavelmente, um resquício deixado por reformas curriculares de décadas anteriores [Vasconcelos \& Borges, 1997].

Nos cursos em que a ênfase recai no estudo dos princípios de medição de grandezas físicas e características de sensores para sistemas de medição, as técnicas de medição, os padrões de medidas, etc. são, em geral, tratados de maneira apenas superficial e sob uma ótica tradicional e anterior a 1990, sem a linguagem do VIM (INMETRO, 2009) e sem as diretrizes do Guia (INMETRO, 1998).

Em ambos os cursos temas como modelagem matemática da medição considerando não apenas o princípio físico, mas também as principais fontes que contribuem para a incerteza da medição, ainda nem despontam no horizonte.

Considerando esta situação, o objetivo deste artigo é o de estabelecer um arcabouço comum que possa servir de base para elaboração de cursos, de graduação ou de pós-graduação envolvendo de maneira integrada os temas instrumentação eletrônica e metrologia. Empregando uma lógica diferente da tradicional, as unidades - disciplinas, módulos, etc. - passam a ser definidas por "resultados do aprendizado", que traduzem as competências que o estudante precisa demonstrar ter adquirido para ser aprovado. Os tradicionais conteúdos aparecem como decorrência das estratégias de ensino/aprendizagem. Ao final são sugeridas atividades experimentais que contribuiriam para desenvolver habilidades práticas e intelectuais do aluno.

\section{PONTO DE PARTIDA}

É amplamente reconhecido que fazer medições para adquirir dados e projetar instrumentos e sistemas de medição estão entre os campos de atuação dos engenheiros na área elétrica. Essa possibilidade era colocada em prática desde os primeiros cursos de engenharia criados no Brasil, quando já podia ser notada a presença de disciplinas com tópicos em medições de grandezas elétricas. Considerando todo o desenvolvimento técnico dos últimos anos e a grande demanda por instrumentos, equipamentos e sistemas de medição, seria lógico supor a existência de consenso sobre os elementos chaves a compor a formação do profissional desta área. A realidade percebida a partir da análise dos currículos de vários cursos no Brasil mostra que a situação é, no entanto, diferente. Uma das razões que levam a isso está no fato de medições serem uma necessidade em praticamente todos os demais campos de atuação do engenheiro, delineando desta forma um sem número de perfis diferentes.

Aliado a isso, deve-se considerar o grande dinamismo da área, provocado por avanços constantes em componentes, dispositivos, técnicas e procedimentos. Além disso, a percepção intuitiva que afeta não só a prática profissional, mas também o ensino, aprofunda vícios e coloca obstáculos difíceis de serem contornados. Certamente a falta de discussões mais amplas entre as partes interessadas contribui para esta situação.

Em vista disso, é de grande interesse descrever um conjunto de conhecimentos e habilidades que definiriam as competências essenciais para a formação de profissionais com condições para atuar com instrumentação e metrologia. Este processo remete, obrigatóriamente, a uma série de questões, tais como:

- do ponto de vista de engenharia, o que, exatamente, é fazer medições?

- qual o significado do termo instrumentação, em engenharia?

- as competências a serem adquiridas pelo estudante, durante o processo de ensino/aprendizagem, para que seja capaz de atuar com metrologia e instrumentação se restringem as habilidades práticas ou conhecimentos e habilidades intelectuais são igualmente importantes?

- existem précondições para o estudante desenvolver essas competências?

- o aprendizado dessas competências pode ser concentrado em uma única unidade de ensino ou seria melhor que ele fosse distribuído ao longo do curso?

- é necessário dispor de uma estrutura laboratorial, que geralmente tem um alto custo, para que o aluno desenvolva as competências necessárias capacita-lo a atuar na área? 
As questões são muitas e as respostas, na maioria das vezes, não são simples. Com o objetivo de contribuir para resolver algumas delas, abaixo são detalhadas propostas para abordagem integrada de instrumentação eletrônica com metrologia.

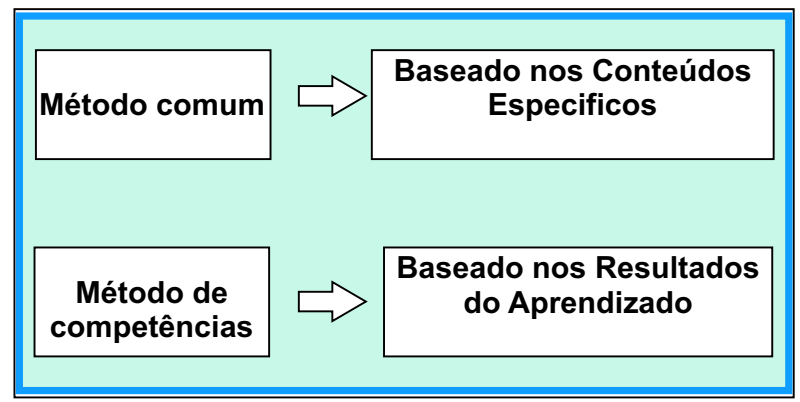

Figura 1: Metodologias normalmente empregadas em cursos de graduação em engenharia.

\section{CURRÍCULOS E UNIDADES DE CURSO}

Os currículos de graduação no Brasil são, em geral, constituídos por unidades de curso elaboradas durante um processo denominado "reforma curricular". Como regra geral, até os novos cursos se enquadram nesse modelo, porque raramente é levado a cabo um processo racional para criação das disciplinas. Em geral se toma algum outro currículo - de um curso mais consolidado - como modelo fazendo ajustes de forma a adaptá-lo as especificidades do corpo docente, sem outra estratégia mais elaborada. As ementas das disciplinas são, em muitos casos, escritas com base em livros sobre o tópico. Essa construção fragmentária, com foco apenas nos tópicos específicos a serem lecionados será aqui denominada de "abordagem baseada em conteúdos" (figura1). Cada unidade é reconhecida por um nome e um resumo descritivo (ementa), cabendo ao professor detalhar os conteúdos. Um exemplo típico seria:

"Instrumentos de medição. Características de desempenho dos instrumentos de medição. Instrumentos e técnicas de medição de deslocamento, movimento, força, torque, pressão, vazão, fluxo de massa, temperatura, fluxo de calor e umidade. Transdução, transmissão e tratamento de sinais. Automação da medição.”.

O detalhamento - programa do curso - fica a cargo do professor bem como as avaliações. Estas, em geral, são realizadas sem um foco claro nos objetivos pretendidos da formação do aluno. O uso do laboratório como espaço pedagógico segue mais uma tradição do que objetivos claros e definidos. O estudante segue uma agenda pré-determinada.

Hoje em dia existe um grande interesse de universidades e mesmo de governos em modernizar o processo de ensino/aprendizado, não só para aumentar o número de estudantes no ensino superior, mas também para melhorar a qualidade do aprendizado [University City of Birmingham, 2009] e aumentar a eficácia dos recursos dispendidos. No caso das universidades brasileiras, isso é essencial.

Unidades de curso - disciplina, módulo, etc. - tais como as voltadas para instrumentação/metrologia podem ser construídas saindo desse lugar comum. Um modelo mais flexível e eficaz foi proposto nos anos de 1970 nos Estados Unidos [Alverno College, 2009] e, na década de 1990, começou também a ser adotado em universidades do Reino Unido. Atualmente é também aplicado em cursos na França, Austrália, India, etc.

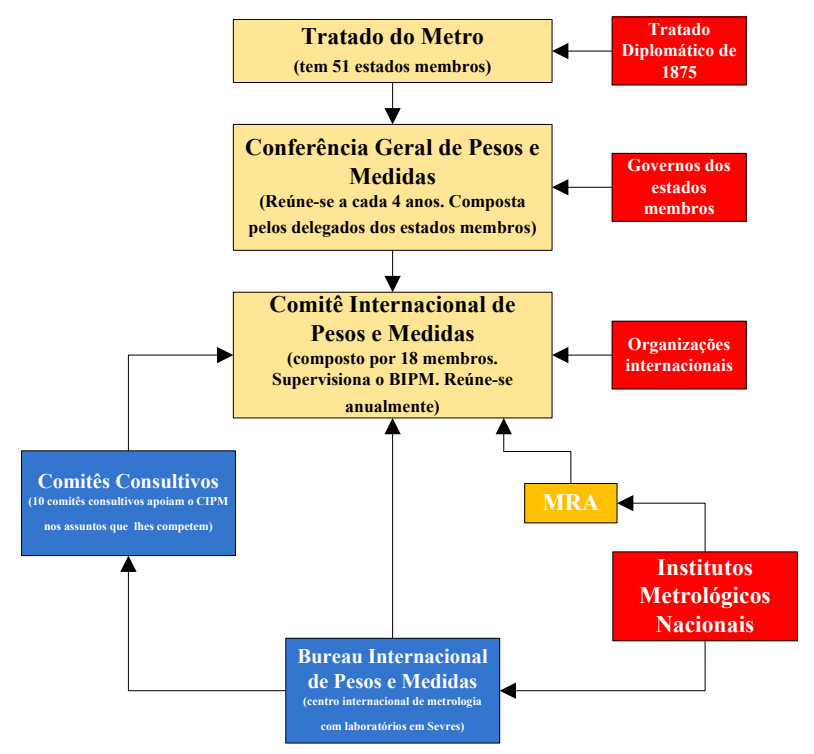

Figura 2: Estrutura do sistema metrológico internacional com base no Tratado do Metro.

\subsection{Unidade de curso definida pelas Competências}

A unidade de curso pode ser elaborada a partir das competências que o estudante tem de adquirir [Voorhees, 2001], ao contrário dos tradicionais conteúdos (figura 1).

Cada unidade de ensino é elaborada para possibilitar ao estudante adquirir um conjunto de competências, compreendidas aqui como os conhecimentos, habilidades ou atitudes, que estabelecem a coerência da formação técnica e pessoal, pretendida. O alvo do processo de ensino passa, então, a ser a verificação da aquisição desses conhecimentos, habilidades e atitudes, que constituem, por assim dizer, os Resultados do Aprendizado [Vasconcelos \& Borges, 1997, Alverno College, 2009]. Para ser aprovado na unidade de 
ensino, o aluno tem, então, de demonstrar os Resultados do Aprendizado (learning outcomes).

\subsection{Elementos chaves de instrumentação - metrologia}

O campo de atuação em instrumentação e metrologia pode ser definido a partir de certos elementos-chave, a saber:

- sistema metrológico (SI, Tratado do Metro, padrões, etc);

- métodos e procedimentos para medição de grandezas elétricas e outras grandezas físicas;

- modelamento da medição, qualidade do resultado (erros, incertezas);

- sensores para conversão de grandezas físicas em sinais elétricos;

- circuitos e dispositivos eletro-eletrônicos de condicionamento de sinais analógicos;

- interface analógico/digital;

- o sistema computacional;

- processamento digital de sinais;

- instrumentos de medição.

As unidades de ensino em cursos de metrologia/instrumentação focadas nos "Resultados do Aprendizado" (RA) podem ser escritas com base nestes elementos chaves, relacionados. Ao invés da tradicional receita, nome/ementa, a unidade de curso fica definida pelos RA que, é importante ressaltar, já contém em sí elementos para avaliação. Exemplos são dados abaixo:

1. (Usar?, Projetar?, etc.) instrumentos para realização de medições de tensão, corrente, resistência, etc. em circuitos CC ou CA (habilidade prática).

2. (Demonstrar?/Criticar? etc.) a relação entre um resultado obtido por medição com correspondentes unidades do SI. (conhecimento/ habilidade intelectual).

3. (Usar?/Elaborar?) métodos e procedimentos para medição de grandezas físicas (temperatura, pressão, vazão, etc). (habilidade prática).

4. (Aplicar?/ Desenvolver?) modelos matemáticos e estatísticos para determinar o resultado completo da medição de grandezas elétricas (habilidade intelectual).
5. (Selecionar?/Projetar?) circuitos e dispositivos eletro-eletrônicos para condicionamento e caracterização de sinais provenientes de dispositivos sensores, para medição de grandezas físicas. (habilidade prática).

A demonstração desses conhecimentos, habilidades e atitudes é o objetivo do processo de ensino. Os "resultados do aprendizado" foram escritos com base na taxonomia de Bloom (1974). Ao escrever os RA acima, no início de cada sentença foram colocados dois verbos. O objetivo é mostrar que um mesmo complemento permite que seja exigido do aluno graus de competência distintos. Já no primeiro exemplo, o nível de habilidade requerida de quem vai apenas usar deve ser bem mais superficial do que o cobrado de quem tem de projetar. Assim, esse mesmo conjunto ou uma parte dele poderia ser usado para definir unidades de curso de nível básico, intermediário ou avançado de graduação e mesmo de pós-graduação, de acordo com o nível de aprofundamento que os autores do projeto curricular desejam dar ao tema. É importante notar que as competências exigidas do aluno devem ser mais básicas no início do curso e mais complexas ao final. É essencial não trocar esta ordem e não se ter uma sequência errática.

Para ter a habilidade prática de usar instrumentos para fazer medições, o aluno deve demonstrar que é capaz não apenas de operar os seus controles, mas também conhecer as suas características e limitações, condições de uso, o significado de estar calibrado, etc.

Para demonstrar a relação entre um resultado de medição com a unidade SI, o aluno deverá aprender que, nos termos da metrologia, resultado quer dizer valor de posição em relação à unidade, acompanhado da incerteza da medição. Isso envolve não somente o conhecimento do VIM (INMETRO, 2009) , do Sistema Internacional de Unidades (INMETRO, 2003) e do Guia (INMETRO, 1998), mas também da estrutura atual do sistema metrológico (figura 2) para que tenha consciência da relação entre aquele resultado, usualmente obtido com um instrumento de medição e a correspondente unidade do SI que o instrumento representa (Albertazzi G. Jr., A. \& Sousa, A.S, 2008).

A habilidade para construir circuitos e dispositivos eletro-eletrônicos, ítem-5 da lista de RA acima, tais como atenuadores, filtros, blindagem contra ruídos eletromagnéticos, conversores AC/DC, conversores A/D, circuitos para linearização do sinal, dentre outros, é muito importante na medida em que esses são elementos essenciais para possibilitar a medição de sinais proveniente de sensores dos mais diversos tipos que afetam diretamente o resultado e a qualidade da medição. 
Medição é uma atividade metódica que requer o conhecimento e uso de instrumentos, de métodos (substituição, diferencial, etc) e de procedimentos detalhados, para que sejam obtidas estimativas do valor da grandeza, com uma qualidade (incerteza) definida.

É comum relacionar o ato de realizar medições com o de registrar as indicações do instrumento com o qual se faz a medição. O engenheiro, no entanto, deve agir de forma diferente, uma vez que o interesse é de realizar medições técnicas. Para tanto, é essencial elaborar o procedimento de medição, que inclui a ação de escrever a função de medição, relação matemática $\mathrm{Y}=f\left(\mathrm{X}_{1}, \mathrm{X}_{2}, \ldots\right)$, entre o mensurando $(\mathrm{Y})$ e todos aqueles fatores que contribuem de forma relevante para incerteza da medição. Para tanto, é preciso conhecer as características de sensores, circuitos, métodos de medição, instrumentos, etc.

O nível de exigência a ser cobrado do aluno é extremamente importante. É interessante ressaltar que um mesmo conteúdo pode ser lecionado para grupos de diferentes níveis. O que distingue o grau de competência é a avaliação realizada.

Outras competências de natureza não-especifícas da engenharia, seriam igualmente importantes, dentre as quais podem ser relacionadas:

a) (Comunicar?/Contrastar?, etc.) os resultados de trabalhos técnicos de maneira clara e coerente (habilidade intelectual).

b) (Organizar?) o ambiente laboratorial visando o bom uso de equipamentos e instalações.

c) (Usar?/Avaliar?) o espaço laboratorial, não expondo a si nem a outros a riscos de acidentes (atitude).

\subsection{Avaliação}

$\mathrm{Na}$ abordagem por conteúdos, o professor elabora as avaliações procurando verificar o domínio do estudante em relação ao tópico específico, tratado na disciplina. Não existe uma estratégia mais ampla para tratar a avaliação que é centrada apenas na disciplina específica.

No caso da abordagem por "Resultados do Aprendizado", um esquema de avaliação deve ser criteriosamente elaborado em razão de sua relevância para se atingir os objetivos pretendidos. O esquema compreende um grupo de métodos de avaliação que são constituídos pelos componentes de avaliação.

O método de avaliação são os meios que o professor lança mão para verificar se o estudante é capaz de demonstrar os $R A$ que adquiriu as competências que definem a unidade de ensino. Estabelece o grau de profundidade em que um determinado RA deverá ser demonstrado.

Os componentes da avaliação referem-se às técnicas elaboradas para avaliar os "Resultados" de uma unidade de ensino. Os componentes podem ser divididos em dois grandes grupos:

\section{- Grupo - 1}

a evidência de que o aluno foi capaz de demonstrar os RA é dada por meios que pressupõem a elaboração ou a análise de textos tais como relatórios de projetos, prova escrita, etc.

\section{- Grupo - 2}

exigem menor dependência da escrita como é o caso dos exames orais, jogos e simulações, projetos e montagens, elaboração de posters, trabalhos computacionais, etc.

Antes de escolher os componentes a serem utilizados é importante verificar se a avaliação é de caráter apenas somativo, para determinar a nota, ou se é formativa, para corrigir equívocos ou aprendizados incompletos. A autoavaliação e a avaliação pelos colegas (alunos avaliando alunos) são também importantes elementos de avaliação. Um outro aspecto diz respeito à confiabilidade (exemplo-1) e validade (exemplo-2) da avaliação (Brown \& Knight, 1994).

Não existe um modelo único. Sempre existe liberdade de escolha para definir o que for mais recomendável para uma situação específica.

Para comprovar que o estudante sabe usar instrumentos de medição, uma habilidade prática, um componente de avaliação adequado seria uma prova prática em um laboratório de eletricidade. O professor deve propor casos nos quais seria demandado o uso do instrumento ou do grupo de instrumentos, de maneira a se comprovar a competência do estudante. Perguntas sobre aspectos teóricos ou práticos dos instrumentos poderiam complementar a avaliação.

Por outro lado, se o RA a ser demonstrado tiver uma maior complexidade, envolvendo um problema em aberto que demande, por exemplo, projetar um instrumento ao invés de apenas usá-lo, o componente de avaliação mais adequado seria um projeto. Uma prova escrita poderia ser usada no caso de apenas parte do conhecimento ser verificado. A demonstração do RA seria na forma de relatório do projeto e de um protótipo do instrumento.

A demonstração de vários RAs pode se dar com um mesmo componente e até no mesmo espaço de tempo. Por exemplo, uma mesma prova escrita pode ser utilizada para avaliar 
conhecimentos, habilidade intelectual e até alguns aspectos de habilidades práticas.

O laboratório é um espaço de ensino que deve ser usado para que o estudante desenvolva também atitudes. As mais importantes dizem respeito às questões de segurança contra riscos de acidentes e de organização para o trabalho experimental. Em ambos os casos, a avaliação pode ser realizada durante uma prova prática, observando concomitamente, o seu comportamento em relação a esses quesitos.

O importante é garantir que o estudante para ser aprovado seja capaz de demonstrar a aquisição das competências que definem a disciplina, fato incomum na tradicional metodologia baseada nos conteúdos.

\subsection{Estratégias de Ensino-Aprendizagem}

São os métodos de ensino/aprendizado empregados para criar as condições para o estudante adquirir as competências e possibilitar que ele venha a demonstrar os RAs.

Para metrologia/instrumentação as competências identificadas envolvem conhecimentos, habilidades práticas, habilidades intelectuais e atitudes. Consequentemente, mais de uma estratégia deve ser usada e isso dependerá também do tamanho da turma, dos recursos disponíveis, das instalações físicas, etc. Entre as estratégias mais comuns estão a aula expositiva, a aula de laboratório, os seminários, o ensino à distância, estudo individual, etc. É importante ressaltar, por outro lado, que não se pretende discutir de maneira detalhada neste espaço, cada uma das possibilidades existentes.

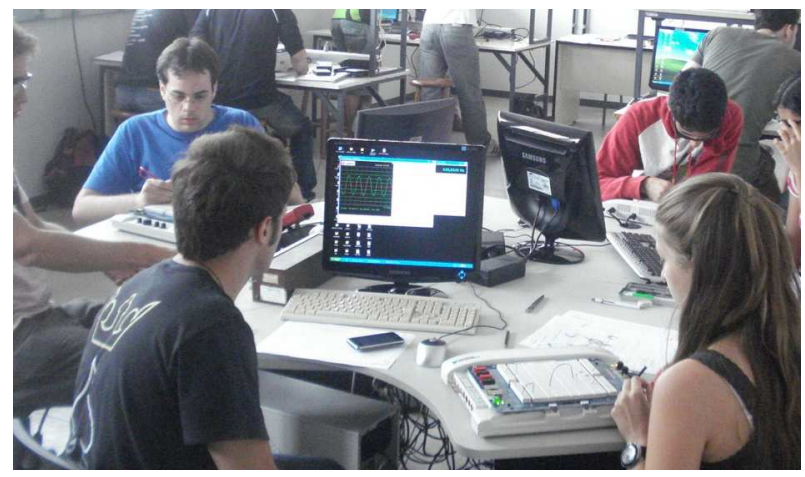

Figura 3: Estudantes de graduação em uma aula de laboratório na UFMG, usando a plataforma $\mathrm{NI}-E L V I S \AA$, da National Instruments Co, em bancada para grupos de 6 alunos.

Em razão de muitos RAs serem classificados como habilidades práticas, o laboratorio se torna um dos mais importantes instrumentos de desenvolvimento dessas competências. Por outro lado, é uma atividade que exige muitos investimentos e, assim, precisa ser bem planejada para cumprir o seu papel.

No laboratório o estudante desenvolve habilidades de uso de instrumentos de medição, elaboração de procedimentos experimentais, atitudes de segurança pessoal e organização no ambiente de trabalho e conhecimentos específicos da disciplina. A aula em laboratório é uma atividade complexa. No entanto, por falta de clareza de propósitos, de planejamento adequado, etc, acaba não atingindo os objetivos pretendidos inicialmente.

Um laboratório equipado para atividades de ensino de graduação e pós-graduação, não possui, necessáriamente, equipamentos muito sofisticados. Já existem várias opções disponíveis para laboratórios na área eletro-eletrônica. Uma solução interessante e moderna é a plataforma educacional NI-Elvis da empresa National Instruments (figura 3).

O nível das atividades em laboratório pode variar substancialmente. Vai desde a demonstração, em que o estudante é um observador da atividade experimental, até a elaboração da tese de doutorado, quando ele age de maneira independente e sem supervisão direta, trabalhando para resolver um problema complexo. É fundamental que ao planejar o curso esta evolução seja considerada.

Sugestões de atividades experimentais, com diferentes níveis de complexidade, para disciplinas em instrumentação/metrologia, são apresentadas abaixo:

- Uso dos de instrumentos para laboratórios de eletricidade, como gerador de sinais, multímetro e osciloscópio. Verificação das suas funcionalidades e especificações metrológicas.

\footnotetext{
Obs. Atividade de caracter introdutório, relacionada com o RA, "usar instrumentos para medição ...”. Devem ser ressaltados os aspectos metrológicos e não apenas a função dos controles do instrumentos. Supõe-se que o estudante já possua habilidades para operações básicas dos equipamentos.
}

- Medição de grandezas físicas (temperatura, pressão, etc) ou elétricas (tensão, corrente, etc) com base em procedimento (INMETRO, 2009) escrito pelo aluno, visando a obtenção do resultado completo, incluindo a incerteza da medição.

Obs. O objetivo é fazer com o estudante aprenda a elaborar procedimento de medição (INMETRO, 2009) para realizar a atividade pretendida 
e mostrar que medir envolve, principalmente, elaborar um modelo matemático (função de medição) (INMETRO, 1998) em que os fatores que mais influenciam a incerteza do resultado da medição devem ser considerados, a partir do qual a qualidade do processo será determinada.

- Dispositivos elétricos e circuitos eletrônicos para minimização da incerteza devido:

- ao efeito de carga.

- à tensão de modo-comum.

Obs. Neste ponto do curso o estudante já deve ser capaz de escrever a função de medição (INMETRO, 1998). Aqui seria importante mostrar que tanto o efeito de carga quanto a tensão de modo-comum precisam ser minimizados no circuito de medição pelo emprego de circuitos amplifi cadores, fi ltros, blindagem, etc., pois caso contrário, mesmo de posse do modelo matemático, a incerteza da medição atingiria valores muito elevados.

- Obtenção por métodos analógicos do valor RMS de sinais variáveis no tempo: a) método do valor médio absoluto, b) método do valor de pico e c) método do valor verdadeiro.

Obs.Objetivo, além de verifi ciar o próprio funcionamento, é identifi car em cada bloco do circuito os fatores que mais afetam a incerteza de medição. O destaque no circuito a) é o papel das tolerâncias dos resistores (Franco, 2002), no b) as chaves analógicas e as características do capacitor e no c) o multiplicador analógico e do circuito integrador (Schnell, 1993).

- Amplificador Lock-In para uso em sistemas de medição.

Obs. Em princípio este é um circuito - analógico, digital ou misto - muito simples. O objetivo da atividade pode ser caracterizar o dispositivo e destacar as suas múltiplas possibilidades de aplicação em instrumentação como eliminação de ruído, obtenção de componentes fasoriais, etc (Schnell, 1993, Tumanski, 2006, Vogelgesang, R., 2004)

- Falseamento (aliasing) e filtro anti-falseamento (antialiasing) para sistemas digitais de medição.

Obs. Aqui dois objetivos podem ser colocados para esta atividade. O primeiro é fazer com que o estudante consiga identifi car o fenômeno do falseamento de frequências (aliasing) e como ele afeta sinais digitalizados, alterando o valor da frequência de alguns componentes do sinal que forem adquiridos sem respeito ao critério de Nyquist. O outro é o projeto do fi ltro AA e como ele pode ser usado, considerando as características do conversor $\mathrm{A} / \mathrm{D}$, para evitar o falseamento em um sistema de aquisição de dados.
- Minimização da incerteza da medição devido a presença de ruídos senoidais: conversor A/D com circuito integrador de sinais.

Obs. O objetivo seria o de mostrar que a digitalização de sinais CC está sujeita a erros devido à presença de ruídos senoidais e que conversores A/D do tipo integradores - rampa, ? $\sum$, etc. podem se tornar praticamente imunes a interferências causadas pela presença de sinais provenientes da rede elétrica, se forem projetados com o devido cuidado.

- Caracterização de conversores A/D para sistemas de medição

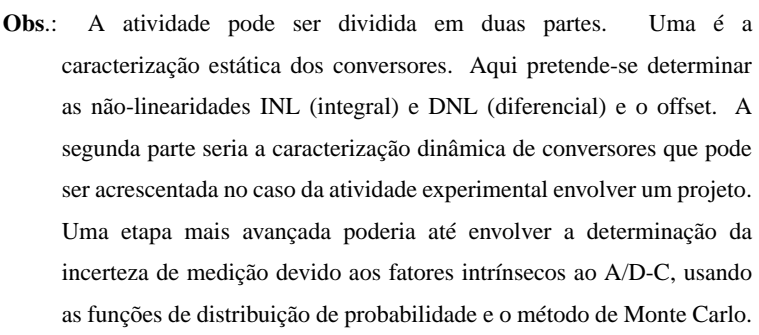

Com base nessas sugestões é possível organizar unidades de curso de instrumentação/metrologia baseado em laboratório tanto para estudantes do início como para os que já estão mais próximos do final do curso. No primeiro caso a participação e o envolvimento do professor devem ser maiores. No segundo, o tema pode ser o projeto e implementação de um sistema de medição, com o professor passando apenas as instruções essenciais e fornecendo o suporte e os equipamentos requeridos pelo estudante. Em qualquer caso, não deve ser esquecido que a atividade experimental precisa ser feita e realizada com segurança

Se a disciplina se destinar a estudantes do início para o meio do curso sugere-se o modelo pedagógico de Resolução de Problemas (Stice, 2009), com o aluno tendo que se preparar para realizar a atividade proposta e a conclusão requeira entregar um produto - circuito funcionando, medições feitas e dados registrados - e elaborar relatório, atendendo a todos requisitos estabelecidos pelo professor. Sempre é importante destacar a necessidade de desenvolver no estudante a capacidade de elaborar o modelo matemático para obter o resultado completo da medição (INMETRO, 1998).

Um último aspecto importante a ser tratado é sobre a bibliografia. Nas Referências são citados documentos (INMETRO, 1998; INMETRO, 2003; INMETRO, 2009) que seriam recomendáveis para qualquer curso na área. As duas últimas são publicações disponíveis sem custo. Para a primeira, até o momento, existe uma versão em inglês (BIPM, 2010). O Guia (INMETRO, 1998) é essencial para compreender o cálculo de incerteza em medições, inclusive 
em casos de modelos não lineares da função de medição, caso tratado no Suplemento-1 (BIPM, 2010). O livro de Albertazzi \& Sousa (2008) é de grande qualidade em questões de metrologia, sendo mais didático e adequado para estudantes dos níveis básicos. O Instrumentação $e$ Fundamentos de Medidas de Balbinot \& Brusamello (2006), é uma referência em dois volumes que trata tanto a parte de instrumentos de medida, volume 1 , quanto a parte de sensores, volume 2. Por último, é importante, lembrar que a bibliografia em lingua inglesa é vasta. Alguns de ótica qualidade foram citados ao longo do texto. No entanto, mesmo essas obras, carecem de uma atualização em linha com os documentos de metrologia também citados.

\section{CONCLUSÃO}

Este artigo teve o propósito de colocar em discussão o foco do ensino/aprendizado para unidades de curso que venham abordar com o equilíbrio e a correção necessária, temas em instrumentação e metrologia, com uma abordagem integradora.

Essa metodologia, já adotada em outros países, as unidades de curso são elaboradas com base nas competências que o estudante deve adquirir para atuar na área. Essa maneira de elaborar a unidade de curso, estabelecendo os "resultados do aprendizado" que o aluno tem de demonstrar para ser aprovado, se mostra mais eficaz do que o método tradicional, de nome de disciplina e ementa/resumo de tópicos, sem nenhum outro direcionamento objetivo, que tem sido adotado em nossos cursos. Ela permite o controle do nível e a avaliação se aluno está, de fato, apto a atuar neste campo. As atividades pedagógicas são elaboradas para possibilitar ao aluno a demonstração das competências.

\section{REFERÊNCIAS}

Ability Based Curriculum, Alverno College, http://www.alverno.edu/ about_alverno/ ability_curriculum.html, consultado em 02/03/2009.

item Albertazzi G. Jr., A. \& Sousa, A.S. 2008). Fundamentos de Metrologia Científica e Industrial. Editora anole Ltda. ISBN: 9788520421161.

Balbinot, A. \& Brusamarello, V.J. (2006). "Instrumentação e Fundamentos de Medidas". Ed. LTC, vol.1 e 2. 2006. ISBN:85-216-1496-9.

BIPM (2010). Guides in metrology: the GUM and the VIM, disponível em http://www.bipm.org/en/publications/

Bloom B.S. et al. (1974). "Taxonomy of Educational Objectives. Handbook 1: Cognitive Domain". Ed. David McKay.
Brown, S. \& Knight, P. (1994) “Assessing Learners in Higher Education", Kogan-Page, London. ISBN 0-7494-1113-9.

Curriculum Design - Learning Components, publicado em http://www.ssdd.uce.ac.uk/ crumpton/ curriculum-design/ default.htm, consultado em 27/09/2010.

Franco, S. (2002). "Design with Operational Amplifiers and Integrated Circuits, $3^{\text {a }}$ edição, Ed. McGraw-Hill Higher Education. ISBN -13 978-0-07-232084-8.

INMETRO (1998). “Guia para Expressão da Incerteza de Medição (ISO GUM)", $2^{\mathrm{a}}$ edição, Serifa Editoração e Informática S/C Ltda., Campinas, SP. ISBN: 85-86768-03-0.

INMETRO (2003) "Sistema Internacional de Unidades SI”. $8^{\mathrm{a}}$ edição, Rio de Janeiro, ISBN 85-87-87090-85-2.

INMETRO (2009) "Vocabulário Internacional de Metrologia - Conceitos fundamentais e gerais $e$ termos associados (VIM)”. $\quad 3^{\text {a }}$ edição, disponível http://www.inmetro.gov.br.

Schnell, L. (editor) (1993). "Technology of Electrical Measurements. Ed. John Wiley \& Sons, ISBN 0471 934356.

Stice, J. E. "Teaching Problem Solving", publicado em http://www.eecs.berkeley.edu/ Courses/ Data/182.html, consultado em 20/04/2009.

Vasconcelos, F. H. \& Borges, M.N. (1997). "Novos Principios e Conceitos do Projeto Curricular para Cursos de Graduação em Engenharia", Revista de Ensino de Engenharia - ABENGE, n. 2, p. 7-33.

Vogelgesang, R.(2004). "Lock-In Amplifier Theory". Encontrado em http://www.nanoopt.org/ images/ stories/ personalNotes/ LockIn.pdf, consultado em 05/11/2009.

Voorhees R (2001). "Measuring What Matters: Competency-Based Learning Models in Higher Education: New Directions for Institutional Research". Ed. Jossey-Bass. ISBN-13: 978-0787914110. 